\title{
Biomanufacturing: from biomedicine to biomedicine
}

\author{
Report from the First Bio-design \& Manufacturing Youth Forum
}

\author{
Yong $\mathrm{He}^{1}$ (1)
}

Received: 18 July 2021 / Accepted: 22 July 2021 / Published online: 24 August 2021

(c) Zhejiang University Press 2021

Recently, the First Youth Forum of Bio-design \& Manufacturing (BDM) was held in Hangzhou on July 3, 2021. Academician Huayong Yang, Editor-in-Chief of BDM, acted as the Chairman and this conference was jointly sponsored by the School of Mechanical Engineering, Zhejiang University, as well as the State Key Laboratory of Fluid Power and Mechatronic Systems. A total of 11 prestigious professors and researchers from all over the world presented at the conference either online or offline, while more than 150 researchers and students from different universities and research institutes attended and heatedly discussed their research in the fields of bio-design and manufacturing from various perspectives.

In the morning session, Professor Yu Shrike Zhang from Harvard University delivered an online presentation entitled Advanced Bioprinting Strategies for Tissue Biofabrication, which systematically introduced the bioprinting methods regarding various tissues or organs. Professor Malin Gou from West China Hospital, Sichuan University, then presented on 3D Bioprinting and Cell-Based Medicinal Products, stressing 3D bioprinting applications in the development of cell-based medicinal products. Professor Xiaobin $\mathrm{Xu}$ from Tongji University presented on Nanopatterning Technology for Biological Applications and Research on Electric Field Driven Motors, explaining in detail recent improvements in nanopatterning and then introduced the issue of how magnetic fields can help with subcellular scale cell detection and controlled drug delivery. Professor Changshun Ruan from the Shenzhen Institute of Advanced Technology, Chinese Academy of Sciences, then presented on the issue of 3D Bioprinting Materials for Tissue Regeneration,

Yong $\mathrm{He}$

yongqin@zju.edu.cn

1 State Key Laboratory of Fluid Power and Mechatronic Systems, School of Mechanical Engineering, Zhejiang University, Hangzhou 310027, China introduced innovated alginate, gelatin, and hydrogel-based bioinks as well as their applications to tissue engineering. Professor Yunhong Liang from Jilin University then reported on Bionic Intelligent Drive Material Design and 3D Printing Manufacturing and introduced bionic robots made of bionic intelligent flexible materials, driven by unconventional driving parts.

In the afternoon section, Professor Jun Zou from Zhejiang University gave a presentation entitled Some Exploration and Thinking on Flexible Drive and Transmission Based on Fluid Power. Professor Zou shared the work of this group as well as perspectives for future research in the area. Associate Professor Zhuo Xiong from Tsinghua University introduced Low Temperature Deposition Manufacturing with Cell 3D printing and systematically reviewed Tsinghua University developments in the field of biofabrication over the last two decades. Assistant Professor Yang Wu from Harbin Institute of Technology, Shenzhen, then presented a talk entitled Study on Soft Tissue Regeneration Based on Structure-Functional Integrated Biomanufacturing and demonstrated biofabrication applications in constricting tissue substitutes, including human cartilage and tendon. Associate Professor Lin Feng from Beihang University then presented about Research on the Application of Field Controlled Micromanipulation and Micro-nano Robot System in the Field of Cell Microsurgery and introduced breakthroughs in field-controlled micronanorobots. Associate Professor Shiyu Liu from the Fourth Military Medical University reported on the Exploration of New Functions of Extracellular Vesicles, which revealed a variety of important biological functions of extracellular vesicles and established biomaterial-based regulatory strategies. Assistant Professor Yan Yan Shery Huang from Cambridge University presented about Cross Length Scaled Biofabrication and specifically introduced a variety of cross-scale biofabrication methods as well as their applications.

Subsequent to a day of discussion, general concerns about bio-manufacturing in the future can be concluded as follows: 


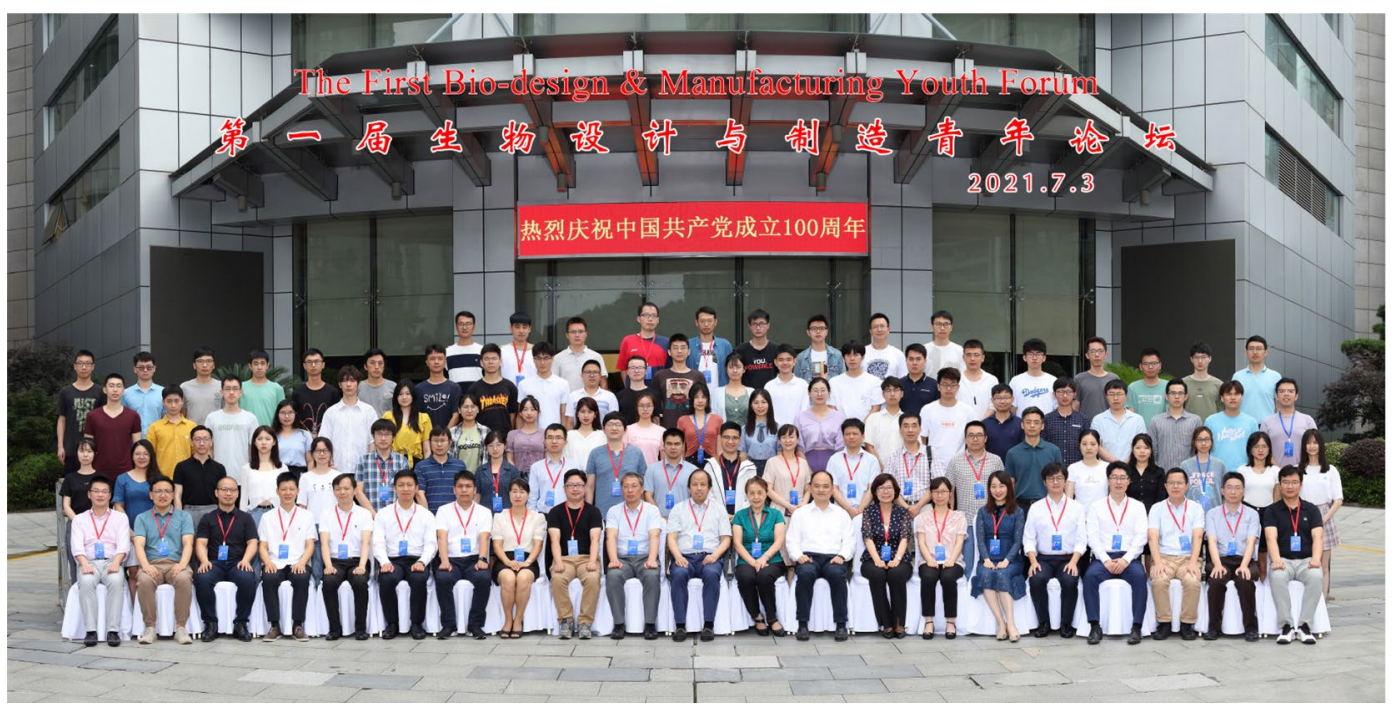

1. What level should biomanufacturing mimic biological tissues? Biological tissues are so complex that one-toone simulation of their macro-, micro-, and nano-structures is almost impossible in manufacturing. This means that in vitro biomanufacturing should focus on imitating the core structure of the corresponding tissue; remaining tissue structure should be completed by the spontaneous growth of the tissue in vivo.

2. Should personalized biomanufacturing link up with standards in medical device approval? Personalized products often introduce changes which means that the industry needs to regulate the requirements of national supervision.

3. What requirements and restrictions are there for cell carrier products to enter the clinic? Cellular status in clinical applications is of paramount importance. Therefore, whether, or not, manufacturing process will affect cell state and lead to cell differentiation is a problem that needs attention. It is therefore necessary to quantitatively evaluate cell damage caused by biological manu- facturing processes such as 3D bioprinting. At the same time, cell carrier products should be further standardized to improve their batch stability.

4. How to mitigate ethical problems? Experiments and developments in biomanufacturing require a lot of clinical data as their basis while access to patient data raises ethical questions. Processes must be regulated, therefore.

5. How to effectively evaluate artificial tissue models? At present, evaluation methods mainly rely on biological detection. However, as technology advances, the effective conversion of biological signals into photoelectric signals will be the future development trend.

These questions will perhaps form the focus of the second forum!

Acknowledgements This forum was sponsored by the National Key Research and Development Program of China (No. 2018YFA0703000). 